\title{
Rassegna di studi su Marco Polo
}

\section{Enrico Vicentini}

Nel corso del Novecento si è assistito a tutto un fiorire di studi poliani (già variamente esaminati da Almagià, Scognamiglio e Surdich) e allo sviluppo di ricerche miranti al pieno riconoscimento della letterarietà del libro di Marco Polo. Solo nel 1928, dopo secoli di fraintendimenti, di faziose interpolazioni, di perentori ragguagli, di aneddotiche informazioni, si giunse, per opera di Luigi Foscolo Benedetto, ad un'edizione compilatoria dell'opera attraverso una collazione di codici. Un ulteriore susseguirsi di ricerche filologiche e di indagini sulla tradizione manoscritta si verificò all'indomani dell'edizione benedettiana e, al tempo stesso, si moltiplicarono le discussioni relative sia alle vicende odeporiche che alle questioni connesse con la stesura, la redazione e la fortuna del Milione. Nel 1924, in occasione del sesto centenario della morte, e poi nel 1954, nel settimo centenario della nascita, ci furono manifestazioni che diedero adito a pubblicazioni di carattere esclusivamente celebrativo. Esse contribuirono notevolmente a restituire a Marco Polo la fama dovutagli e ad attribuire validità e significato alla sua opera letteraria.

Nel Novecento, con l'espansione dei mass media, Marco Polo personaggio e le sue vicende sono ulteriormente passati al cinema, al teatro, alla televisione. A tali testimonianze spesso non si pud attribuire alcun valore per la mancanza di termini di raffronto: ${ }^{1}$

il cinema ... s'è offerto di darcene [del Milione] una immagine fantastica e contemporanea a noi, tesa sul filo dell'avventura, e non ha cercato nell'iconografia o nella approssimazione una qualunque probabilità di vero. (Giani xxi)

È del 1982 una trasposizione televisiva, un kolossal che ha provocato numerose controversie e polemiche sulle pagine di quotidiani e periodici, ${ }^{2}$ ma che ha il pregio di aver rinnovato l'interesse di un'ennesima generazione per le tematiche poliane, e di aver permesso che

il racconto e il personaggio di Marco Polo ... venissero a inserirsi con nuova ed icastica vitalità nella cronaca giomaliera, fatta veicolo, insieme alle immagini del piccolo schermo, di emozioni e interessi che il mondo orientale, le sue terre, $i$ suoi popoli, $i$ suoi usi sono ancora capaci di suscitare. (Ruggieri $\mathrm{ix}-\mathrm{x}$ )

Nello stesso periodo vi è stata una fioritura di libri su Marco Polo, Venezia e l'Oriente,' e sono uscite numerose ristampe del Milione, nonché rimaneggiamenti volti a rendere il testo alla portata di tutti. Nel corso dei secoli l'interesse per l'opera poliana non è mai diminuito e negli ultimi anni è avvenuta una moltiplicazione di iniziative di vario genere. 
Dopo l'articolo di Franco Borlandi in cui vengono studiate a lungo le caratteristiche di composizione, di materiale e di stile che accomunano Le divisament dou monde poliano e la Pratica della mercatura di Francesco Balducci Pegolotti, e dopo la ristampa dell'edizione del Milione curata da Ettore Camesasca in cui si riprende il riferimento benedettiano della responsabilità degli amanuensi che "non si sentivano obbligati a una fedeltà troppo rigida" (22) al testo, si colloca innanzi tutto l'apporto di Cesare Segre che inserisce il libro poliano nel contesto storico dell'Italia settentrionale divenuta centro di raccolta di poemi cavallereschi, di fenomeni collettivi di carattere sociale o guerresco-feudale e di opere in prosa che rispondevano a necessità pratiche, il che tendeva a condurre alla letteratura divulgativa (Lingua 14-19).

Segre considera la fisionomia del Milione frutto della fusione di varie aspirazioni, come la letteratura descrittiva "che segna un primo avviamento alla curiosità scientifica ed etnografica"; perciò l'opera è da considerare "punto di convergenza di numerose tradizioni" (27) e risultato di attività pratiche, di esigenze della fantasia e di finzioni romanzesche. Nel Milione l'inchiesta diretta "rafforza e contiene il lungo nastro di fantasie e travisamenti pseudoscientifici di cui pure essa costituisce un punto di arrivo" (28); infatti risaltano i dati frutto di esperienza diretta e la sembianza da memoriale dai tratti novellistici e epici. Nella storia del trattato scientifico il Milione si configura fra quelle opere che non sono frutto "di una conoscenza mediata del mondo" (28) perché si allontana notevolmente dalla prassi compilatoria medievale intrisa di superstizione e simbolismo; il Segre, nel sottolineare l'importanza dell'influenza proveniente dalla Francia, rileva che si importarono da quei "prossimi mercati letterari ... non solo le compilazioni didascaliche ... ma anche le invenzioni cavalleresche e cortesi così profondamente radicate nel nativo terreno ideologico" (29).

$\mathrm{Si}$ importò anche la lingua francese che aveva largo corso e che, nel $\mathrm{Mi}$ lione, è "veicolo d'elezione d'un genere letterario", come sottolinea Aurelio Roncaglia (730-31). Nel trattare la materia della letteratura franco-veneta, il Roncaglia accentua il valore che ebbe in particolare la narrativa epico-cavalleresca nella fortuna dell'antica letteratura francese in Italia e sostiene che la fenomenologia linguistica che caratterizza il franco-veneto non è dovuta né ad una realtà dialettale né a copisti e scrittori, ma all'esperienza comunicativa orale che è intenzionale: perché fosse recepita dal pubblico ci doveva essere un abbassamento linguistico. Si potrebbe allora aggiungere che la presenza di venetismi nel Milione non è necessariamente dovuta all'insufficiente conoscenza del francese da parte di Rustichello o di altri amanuensi posteriori (738), ma che allo stesso modo in cui nelle opere tradotte si doveva mantenere un certo prestigio letterario che ricordava l'atto d'importazione, anche in quelle originali era necessario garantire "la pratica comprensibilita da parte d'un largo pubblico locale, che nella sua maggioranza non aveva, né poteva avere, conoscenza adeguata della lingua francese, anche se ambiva ostentarne l'intelligenza" (740). Il Milione venne così a circoscriversi entro i confini 
di una tradizione che, pur comprendendo anche opere di genere diverso, ha come aspetto più conosciuto e diffuso quello romanzesco.

In un successivo studio di Cesare Segre si ha un'analisi della didattica scientifica nel cui ambito si configurano fra l'altro enciclopedie, con fine divulgativo, farcite di descrizioni geografiche delle meraviglie del mondo e di notizie scientifiche, a volte integrate in un sistema teologico ("Le forme" 134-37). Per quanto riguarda le compilazioni geografiche, sempre nell'ambito della didattica scientifica, il Segre accentua l'aspetto fantastico della Lettre du Prêtre Jean, del Romanzo d'Alessandro e delle missioni di Giovanni dal Pian del Carpine e di Guglielmo di Rubruck, le cui spinte alla conoscenza dell'Oriente rappresentano, per lo studioso italiano, "le esperienze e gli ideali" compresi nell'opera poliana. Per Segre il Milione è "nello stesso tempo una relazione di viaggio e una descrizione geografica" ("Le forme" 142), ma si potrebbe osservare che il libro del Veneziano rientra in tutti i campi della didattica scientifica, perché circoscrivendolo soltanto in una di tali aree ne viene automaticamente negata la natura composita.

Nell'analisi della letteratura franco-italiana compiuta da Antonio Enzo Quaglio un capitolo a parte viene dedicato al Milione, opera da collocare tra cronaca e avventura, decisamente classificata come "libro romanzo" (329) per la collaborazione con Rustichello che fa permanere una situazione di incertezza relativa anche agli schemi narrativi utilizzati. Marco viene raffigurato come mercante e memorialista, biografo ed esploratore; ciononostante "il libro non è solo il resoconto di un mercante stilato con intenti pratici, né tanto un documento geografico o una relazione di viaggio vergata con intendimenti scientifici". Il Milione non si adatta bene alla collocazione entro lo schema convenzionale impostogli perché "la sua orbita trascende gli scopi documentari, i gusti didascalici, le preferenze pedagogiche del secolo" (330). L'opera poliana viene a rappresentare un atlante preciso che si indirizza ai lettori più disparati: da coloro interessati al commercio, alla scienza e quindi alla conoscenza, a coloro che sono alla ricerca del fantastico e del meraviglioso.

Con gli anni Settanta si ha un rilancio di studi poliani volti alla pluritestualità e alla critica testuale. Nella "Prefazione" all'edizione Adelphi, Valeria Bertolucci Pizzorusso si sofferma sul ruolo di auctor di Marco:

il suo contributo non deve essere stato, in fase di stesura, esclusivamente orale, preceduto soltanto dal lavoro di recupero, nel tesoro della memoria, delle "cose da ricordare", trascelte tra quelle che non sono ritenute degne o opportune. (xii)

Gli effets de littérature creati da Rustichello per smorzare "un poco la sensazione di verità che scaturiva ... dal racconto" (xiv) ne aumentavano, al tempo stesso, la credibilità. La sequenza letteraria utilizzata da Rustichello faceva del libro di Marco Polo un insieme di romanzo, manuale mercantile, trattato di etnografia, itinerario, relazione diplomatica. Tutto ciò porta la Bertolucci Pizzorusso ad asserire che questo libro, "tanto vario e composito" (xiii), 
presenta una "imbarazzante complessità riluttante ad un inquadramento di genere" (xiv): la forma letteraria del romanzo risulta quindi troppo riduttiva.

È necessario tenere conto dei trascrittori e dei lettori; per dimostrarne l'influenza sul testo la studiosa porta come esempio la versione toscana, dalla quale si ricava una lettura "orientata sui dati commerciali e sugli aspetti novellistici" che "collima perfettamente con gli interessi e i gusti" del "ceto borghese mercantile ... proprio allora in grande espansione" (xv). Quindi se il Milione era, nella fase originaria, in un "involucro romanzesco", esso successivamente si venne a collocare fra gli scritti mercanteschi allora in voga, "ampliando il già ricco ventaglio dei tipi prosastici in antico toscano" (xvii). Infatti la versione latina e quella veneta appartengono alla prima fase di diffusione del libro e un confronto fra le due "permetterebbe interessanti e motivate osservazioni sui diversi ambienti ricettori, che orientarono il testo secondo le rispettive attese, mutandone i contrassegni di genere" (xviii).

Già il Contini aveva affermato che il Milione appartiene a pieno titolo al franco-italiano, per "la varietà di contaminazione idiomatica"; in questo caso tuttavia si dovrebbe andare oltre la veste linguistica perché l'opera poliana è permeata di una "energia extratestuale" che ne fa "appunto un canovaccio di perenni traduzioni e successivi adattamenti" e anzi, possiamo dire, di molteplici letture e di molteplici intepretazioni.

La componente geografico-mercantesca viene messa in risalto da Ugo Tucci per il quale il libro di Marco Polo, come era già stato osservato varie volte, non solo "segna una svolta decisiva nella letteratura geografica sull'Asia" (641), ${ }^{3}$ ma ha anche alla base l'idea del manuale di mercatura. Egli osserva che il Veneziano non avrebbe potuto fare diversamente:

Marco Polo ha ordinato le sue esperienze di viaggio secondo la struttura del prontuario di tecnica commerciale non col deliberato proposito di comporne uno con fini pratici veri e propri, ma perché era la forma nella quale un mercante era abituato a veder rappresentato il mondo. (643)

Più studiosi si sono già soffermati a considerare quanto sia rilevante, ai fini della definizione della veste che deve assumere un testo, l'influsso dell'ambiente che commissiona la trascrizione ("a Venezia queste composizioni nella seconda metà del Duecento erano già largamente diffuse" [643]). Secondo il Tucci si deve invece evitare di fissare l'immagine del testo secondo un "tipo professionale e sociologico"; esso va inquadrato nell'"ambiente mercantile veneziano al quale appartiene e alla cui luce va interpretato" (643). Nonostante la rielaborazione di Rustichello, nel Milione si conserva la struttura del manuale di mercatura che attribuisce "un ordine sistematico all'esposizione" (654), perché in tal modo il testo si presta ad accogliere tutta una vasta gamma di notizie. Il vero viene fuso con le "tradizioni fantastiche inveterate" che "avevano l'ufficio di avvalorare la narrazione", sicché dal racconto si ricava "una credibilità maggiore di quella che poteva spirare da una verità sovvertitrice" (658). 
Se il testo poliano è stato condizionato dall'aspetto mercantile, senza comunque indirizzarsi esclusivamente ad un determinato gruppo di lettori, la Bertolucci Pizzorusso insiste invece sulla "mancanza di mediazione di un genere letterario ben definito", il che determina la "problematica fisionomia linguistica originaria" dell'opera (Enunciazione 5). Il testo poliano, prosegue la studiosa, viene indicato come livre per più di cinquanta volte e col titolo Divisament dou monde "di tipo . . . 'oggettivale' . . . . [L'autore] intende inserirlo nel genere già tradizionale del trattato enciclopedico piuttosto che in quello dei ricordi personali o della relazione di viaggio" (10) in cui si sarebbero narrate piuttosto le vicende particolari del protagonista. Commentando le ipotesi del Borlandi, la Bertolucci Pizzorusso osserva che se si considera il francese "parlato" di Marco e quello "della scripta romanzesca" di Rustichello, si nota che varie esigenze vengono conciliate, e si ha una convergenza di vari generi "proprio in vista di quel pubblico universale individuato e apostrofato dal vos di apertura" (14). Non è possibile, quindi, delimitare la "destinazione dell'opera alla ristretta cerchia mercantile" (14), come il Borlandi aveva proposto.

La sistemazione di genere per il testo poliano non è impresa facile, ma già nell'esordio ci si trova davanti ad un modello di prosa narrativa e enciclopedica, di stampo medievale, ${ }^{4}$ in cui si mette in rilievo

la sproporzione tra la scarsezza delle garanzie offerte e l'esigenza di veridicità di un testo a carattere referenziale di autore sconosciuto. Data la difficoltà della verifica dei contenuti, non resta che puntare sull'attendibilità del responsabile ed assicurarsi cosi la fiducia del destinatario. (20)

Nel prologo viene tracciata la figura morale di Marco Polo e quei capitoli vengono a rappresentare "l'atto autobiografico" come atto di autentificazione; tale prologo connota seppur in grado ridotto la descrizione poliana e "accoglie solo i materiali funzionalizzabili" (21) al fine del convincimento e della persuasione.

Nel libro della descrizione del mondo si possono identificare vari livelli: il primo è quello del discorso con l'uso del "presente cosiddetto atemporale della descrizione geo-etnografica", mentre il secondo "si avvale delle modalità narrative temporali propriamente del passato ... sul quale si dispongono la 'storia' e ... diversi elementi narrativi (accenni a situazioni politiche precedenti, aneddoti storici e leggendari, episodi vari e novelle)"; gradualmente, si arriva ad "un tempo psicologico che tende a confondere le due diverse cronologie della 'storia' e del 'discorso"' (23-24). La complessità della questione viene quindi rappresentata dalla compresenza di due sistemi: "quello della biografia (in cui l'io del 'discorso' è diverso da quello della 'storia') e quello dell'autobiografia (in cui essi coincidono)" (29).

Dall'archivio della memoria l'elaboratore del libro ha dovuto scegliere "l'ordinamento e la distribuzione del materiale memoriale che lo costituisce" (30). Quel materiale concernente l'esotico non viene inserito integralmente 
per l'aspetto superficiale dell'eccessiva diversità di certi costumi; si sceglie piuttosto in base all'utilità e ne risulta l'"attivazione del ruolo del narratario nella scelta e nel taglio del materiale da testualizzare" (31). Un criterio di utilità si formalizza nella "preoccupazione di smentire credenze false e dannose presupposte nel narratario" (32), come nel caso della salamandra, dell'unicorno, delle scimmie imbalsamate, dei grifoni e via dicendo. Si procede, quindi, con "la decisione di legare la 'descrizione del mondo' allo schema dell'itinerario" (35) che non deve seguire un determinato ordine perché viene svolto ad esperienza compiuta. Vengono raggruppate schede da manuale di mercatura, frutto di segmentazione, affinché "ad ogni unità testuale corrisponda una stazione del viaggio: si ha così la mappa del mondo, in figura di atlante ragionato" (37). Nel libro-atlante ogni unità testuale si apre con il nome della precedente: l'assenza di tale enunciazione è "indizio di una variazione di genere del contesto nelle direzioni della 'storia' o della 'novella' . . . , della descrizione di battaglie, della trattatistica in senso proprio . . . che si realizzano in enclaves di vario tipo" (37-38) le quali superano generalmente l'unità media e si protraggono per vari capitoli. Secondo la Bertolucci Pizzorusso, "la novella, la descrizione nella 'descrizione', la monografia non sono altro che soste o meglio tappe più rilassate e confortevoli dei viaggiatori"; vi si configura una struttura che "presiede al sistema compositivo dell'opera come trattato enciclopedico". La struttura libresca che si sta descrivendo è frutto della

esigenza propria del dettato letterario di autoorganizzarsi per argomenti, secondo principi di logica interna, formali e/o funzionali che siano, liberi comunque da obblighi verso una linea strutturante esterna, referenziale, quale è quella che lega la relazione di viaggio all'itinerario reale. L'indice del libro infatti non ne rappresenta soltanto la topografia. (38)

Nella terza sezione, secondo la suddivisione inaugurata dal domenicano Francesco Pipino da Bologna, traduttore trecentesco del libro poliano, all'interno dell'opera sono inseriti altri testi: il libro sull'India, l'excursus sul Giappone, il trattato sul Gran Cane, la trattazione dei Tartari che nell'insieme "dimostrano la possibilità di una sovrapposizione dell'enciclopedia all'itinerario" (41). Il libro nel libro attorno al Gran Cane "modifica i rapporti tra la narrazione e lo schema referenziale che la subordina" in quanto la prosa tipicamente rustichelliana dimostra "una perfetta rispondenza dell'enunciato ad un modello standardizzato e lucido" (42). Nel saggio della Bertolucci Pizzorusso si ha il primo tentativo di lettura del libro di Marco Polo che superi i canoni della critica testuale. La studiosa è andata oltre l'ortodossia che ha caratterizzato la storiografia poliana precedente, per arrivare ad una lettura che tiene conto delle teorie critiche letterarie più avanzate.

A tale proposito di particolare interesse risulta il saggio di Antonio Carile che affronta il tema dei criteri scelti dal Veneziano per l'esposizione dell'ambiente. La questione circa la collaborazione tra Marco Polo e Rustichello 
non può non essere menzionata perché il contributo dell'estensore "configura emblematicamente e quasi materializza il rapporto dell'autore con il pubblico cui Le divisament dou monde è rivolto": la tradizione testuale, la fortuna del libro che ne derivò costituiscono "uno degli esempi più significativi dell'interazione fra autore e destinatari del testo" (19). L'indagine del Carile ricalca le tesi formulate precedentemente e mette in rilievo formule-soffermandosi sui resoconti, le cronache, il romanzo, il manuale di mercatura-senza comunque nessun tentativo di raggruppamento enciclopedico.

Pierre-Yves Badel riprende nel suo saggio le tesi della Bertolucci Pizzorusso, tenendo in considerazione soprattutto le meraviglie, le cui fonti vanno cercate nella storia e nella geografia medievali. La narrativa dell'opera segue due tematiche: una personale-quasi autobiografica nel caso dei racconti del viaggio del padre e dello zio di Marco Polo, e del loro secondo viaggio con Marco - l'altra collettiva. La prima è rappresentata dai diciannove brevi capitoli iniziali che costituiscono un libro "hors livre" da cui si apprende che l'autore "ressourtit à un autre genre littéraire" (9). La tematica della storia collettiva si manifesta dove "la merveille a parfois le caractère d'une anecdote, d'un miracle, du récit des batailles qui mirent aux prises les Tartares" (10): è ovvio che qui l'autore ha presente dei modelli letterari che si rifanno all'agiografia, al romanzo e all'epica. Il Badel conclude che

la Description du Monde n'est pas un récit de voyage, elle appartient à un genre typé, celui des compilations de notices geographiques qui constituent le tout ou une partie d'ouvrages dérivant de l'Histoire naturelle de Pline. (10)

Lo studioso francese non mette in discussione le notizie redatte da Marco Polo, ma si sofferma sulla meraviglia che è "une de ses notations [qui] s'amplifie en une digression narrative ou descriptive". Il Badel prosegue affermando che, sebbene ciò sembri paradossale, "la merveille est un topos de l'ouvrage ethnographique" e assegna credibilità alle descrizioni perché essa e "le réel de l'autre" (11).

Svolgendo un'analisi della dinamica del meraviglioso, il Badel ha assegnato una nuova prospettiva al legame esistente tra il meraviglioso, l'opera poliana e l'enciclopedia. Al tempo stesso lo studioso francese ha dimostrato che con il distacco del Veneziano dai racconti popolari e leggendari si esce dalla sfera dell'enciclopedia tradizionale per inoltrarsi nell'enciclopedia come fonte di notizie verificate tramite l'esperienza diretta. Questa è la premessa a quanto affermato da Marcello Ciccuto: Marco Polo ha redatto la propria opera forse perché era munito dell'esperienza diretta che il monaco Cosma Indicopleuste affermava fosse di primaria importanza per "dissipare la retorica intellettuale, vera e unica responsabile della circolazione di leggende fantastiche sulle zone meno conosciute della terra" (Introduzione 13). Successivamente il Ciccuto si sofferma sui pellegrinaggi in Terrasanta ("che non ebbero ... per la maggior parte il conforto della relazione scritta" [13]), sulle cosiddette relazioni di viaggio degli Arabi Mas'oudi e Idrisi, sui rac- 
conti delle crociate, sul Giornale di viaggio della monaca Eteria, e sottolinea che la "compenetrazione di cronaca, memoria e dato religioso-letterario è un tratto usuale per testi di questo genere" (14), anche se in essi la fantasia viene talvolta a supplire alla scarsa disponibilità di dati.

Il mito dell'Oriente, "consegnato al grosso pubblico dal ciclo romanzesco delle Storie di Alessandro" (16), si espande per tutto l'Occidente e viene ripreso nel Milione, con l'aspetto di un prontuario mercantile. Al tempo stesso il Ciccuto sottolinea che il libro non è un "rigido accumulo di notazioni analitiche sulla realtà economica dell'Asia" (33) data la collaborazione tra Marco Polo "computista" e Rustichello "colto scrivente" (32). L'esperienza diretta viene concretizzata nel Milione mediante un equilibrio tra realismo e tradizione, e la struttura enciclopedica viene a rappresentare il "paradigma della mentalità medievale" (34).

Giorgio Manganelli, autore che predilige quella letteratura che possa determinare "l'elaborazione della sua stessa poetica" ("Marco Polo" 163), e critico che rielabora la "coscienza del carattere di 'infermità', di 'anomalia', di faticosa 'magia' proprio della letteratura" (Ferroni 10057), ovviamente imposta la sua lettura del Milione in una diversa prospettiva: "l'itinerario di Marco Polo è una istoria, una invenzione veridica ma tutta mentale di qualcosa che esiste non perché è sperimentabile, ma perché è raccontabile e materia di ricordo". Nel Milione Marco Polo non fa trasparire l'eccitazione del narratore ma "la pazienza di qualcuno che è intento a disporre il regesto di un mondo" ("Marco Polo" 163) e pertanto nasce un libro di innumerevoli tracce, indizi, allusioni invitanti.

Le molteplici sensazioni che possono stimolare il lettore a seguire svariati itinerari-in quel labirinto in cui ad ogni angolo si nasconde una sorpresaparagonabile alla serie di trasformazioni, alle edizioni e alle traduzioni che ha avuto l'opera stessa. Come giustamente scrive il Manganelli, "questo libro infinitamente letto, tradotto in tutte le lingue possibili, tradotto sempre da altre traduzioni, si è rifiutato di esistere nell'originale" ("Marco Polo" 164) e divenne frutto di varie possibilità "di favola, di fantasia, qualcosa che esiste solo nelle innumerevoli varianti, di cui non è lecito tentare una edizione definitiva" ("Marco Polo" 165): e in tal modo viene riconosciuta la struttura labirintica dell'opera poliana.

Il Manganelli tende a mettere in rilievo il carattere fantastico di tutta la letteratura, anche di quella solitamente considerata realistica, e a darle la dimensione di congegno artificiale, di giocattolo meccanico (Ferroni 10059). Essa

ambisce a partorire il libro onnicomprensivo; un libro capace di generare infiniti libri, capitoli ingegnosamente solidali, classificazione e descrizione di ciò che non esiste. ("Letteratura" 50)

L'Asia di Marco Polo, prima di essere descritta, "ha voluto essere uccisa" ("Marco Polo" 163): per Manganelli è infatti la morte che motiva la scom- 
parsa del libro che "voleva 'morire', perché . . . doveva moltiplicarsi in innumerevoli testi 'quasi' esatti" che avrebbero generato "libri fatti per il lettore, per il copista, per il traduttore" ("Marco Polo" 164).

Egli mette sullo stesso piano i ruoli del viaggiatore e del lettore perché ambedue devono "attraversare segni, immergersi in volumi fitti e brulicanti, popolati di scritture mobili, di disegni dotati di respiro": anche il mondo "è un immenso libro, che il lettore percorre riconoscendovi incastri, sovrimpressioni, spostamenti di segni, artifici" (Ferroni 10079). Secondo Manganelli il lettore del Milione ha la sensazione che il libro gii stia continuamente sfuggendo di mano, perché "è un libro irrequieto e instabile, un libro errabondo come fosse favola, sebbene sia fedele trascrizione di una vita impossibile ma reale" ("Marco Polo" 165).

L'uccisione del libro va attribuita a Rustico che "tentò di adulterare, di insinuare la favola in quel libro geniale ma ignaro di astuzia" ("Marco Polo" 166). Allora il Milione preferì "dissolversi in innumerevoli falsi quasi veri" ("Marco Polo" 166). Verso la fine del Milione, Rustico riuscì ad inserire "qualche frase del suo repertorio" ("Marco Polo" 167) e l'opera poliana "venne lett $a$ come nuova favola ... favola tanto nuova, da non lasciar fiato alle favole antiche" ("Marco Polo" 169).

Il fascino del viaggio-lettura si esplicita, come scrive il Ferroni,

nel fatto che essa [la condizione del viaggiatore lettore] non permette mai una identificazione totale col testo, lasciando un margine di non conosciuto, di non riducibile ai metri e ai metodi del lettore, per quanto perfezionati e attenti possano essere. (10079)

E così, come sottolinea il Manganelli, il Milione non può mai essere collocato entro una tradizione ben determinata: "questa rinuncia stilistica restituisce all'autore, al viaggiatore le sue dimensioni casualmente e definitivamente grandiose" "Marco Polo" 169), quindi il libro morto è "un libro perduto da sempre e da sempre con noi" ("Marco Polo" 173).

L'operazione letteraria svolta da Marco Polo viene analizzata attentamente nell'opera manganelliana: il Milione viene in tal modo a configurarsi nell'ambito della letteratura fantastica oggetto dell'indagine dello scrittore come un universo impossibile ma compatto, organizzato e irrefutabilmente argomentato ("Letteratura" 45-46). Si potrebbero avanzare ipotesi sui capitoli che occorre sollevare come "le botole delle parole, per scoprire altre botole" e arrivare al "palinsesto universale". In lettore deve farsi "talpa, rettile, formicaleone, e scovare tane, scavare cunicoli, finché tutta la creazione sia un prezioso e fragile termitaio di parole" ("Letteratura" 49). La metafora del vaso di Pandora, applicabile alle parole, alle suddivisioni, va pure riportata al metro e al metodo letterario utilizzato dall'autore/estensore; quindi detta pluridimensione identifica il Milione stesso come elemento fantastico.

Secondo Umberto Eco l'opera poliana va inserita "in una serie di racconti enciclopedici" (157) e, giacché quella tradizione si basa su opere scritte a tavolino, senza che l'autore abbia vissuto le avventure descritte, si potrebbe 
affermare che "il libro di Marco Polo anticipa un genere" (157). Infatti il Veneziano è piuttosto redattore di un reportage, il che comunque dimostra che Marco era stato influenzato dalla "cultura delle enciclopedie medievali" le cui informazioni provenivano spesso "per lunghi tragitti storici, dal leggendario orientale" (159). In fin dei conti Marco "è uomo del suo tempo e non riesce a sottrarsi all'influenza di quei libri-magari non letti-che gli insegnano cosa dovrebbe vedere" (159). Anche se nel Milione mancano l'allegoria e i moralismi, aspetti tipici di altre enciclopedie medievali, i contemporanei di Marco Polo leggevano il libro con gli stessi criteri adottati per i suoi "predecessori fantasiosi" (165).

Nella recente introduzione di Cesare Segre all'edizione a cura di Gabriella Ronchi, a Marco Polo "viaggiatore e avvezzo a resoconti amministrativi e dispacci diplomatici" (xi) viene categoricamente riconosciuto il ruolo di autore, "proprio perché alla sua autorità (autore-fonte e testimone attendibile) si fa appello spesso, nel libro" (xii). Quindi ne risulta un Marco Polo che "si fa avanti e pare esibisca il libro dei conti che ha avuto occasione di compilare" (xiii). Dopo aver delegato a Rustichello la stesura "e l'oggettivazione nel libro (messaggio reso autonomo dal destinatore), Marco Polo si presenta, persona dentro al libro, viaggiatore precedente il resoconto del viaggio e fideiussore della sua verità" (xiii). A ciò fa seguito una compilazione di notizie apprese per esperienza diretta e di altre tolte da "testi scritti" che l'autore "aveva letto per prepararsi al viaggio o per confrontarli con quanto vedeva con i suoi occhi" (xiv). Le pagine dei libri che, secondo Segre, "l'avevano incitato e preparato" assegnano un carattere di legittimità alla "fiaba del suo viaggio", mentre la fusione di fonti autentiche e possibili e della componente letteraria aggiunta da Rustichello è stata saturata "alternativamente nella pragmatica della fruizione"; Marco "dispenserà secondo i casi le illecebre del meraviglioso e i preziosi suggerimenti di un grande viaggiatore ad altri che ne seguiranno le orme", facendo nascere quella tradizione del Milione caratterizzata da "una inesauribile attività rielaborativa, che ogni volta dà un tono diverso alla voce riferita da Rustichello" (xv).

Le lingue in cui l'opera poliana fu tradotta ne dimostrano la notevole fortuna. Infatti, per diffonderla più rapidamente, i traduttori e i copisti la volsero "nella lingua ritenuta più efficace per la sua ulteriore propagazione", vale a dire il latino per missionari e scienziati, "le lingue volgari e i dialetti per commercianti e viaggiatori, ma anche per un consumo voluttuario, da libro di evasione"; e altre stesure che accentuano vari "aspetti (documentario, storico-geografico, antropologico, diaristico, fantastico) che già vi sono presenti" (xvii).

Sia per il titolo che per la struttura, il Milione si inserisce nel genere del trattato geografico che, a detta di Segre, si rifa alla nobile tradizione dell'enciclopedia. Il trattato geografico, precedentemente basato su materiali ricavati da altri libri e intriso di notizie fantasiose, viene ravvivato dal Veneziano il quale "si collega, differenziandosene, con i vari 'romanzi' d'Alessandro"; 
anch'egli si occupa di mirabilia, "non però come i produttori di letteratura pseudoscientifica", gli autori di enciclopedie, "bensì come colui che, entrato in dimensioni già in sé eccezionali, misura ogni tanto gli scarti, anche nelle credenze e nelle usanze, rispetto alle norme del mondo a lui noto" (xxii).

La classificazione di trattato geografico è stata assimilata da Segre con quella dell'enciclopedia perché "il trattato geografico funge da contenitore molto pratico per tipi di testi alquanto vari" (xxiii): storia, exemplum, racconto agiografico, racconti di carattere novellistico e via dicendo. "È chiara la segnaletica per questo programma di moltiplicazione dei generi" (xxiv) che conduce alla varietà stilistica rappresentata dai dialoghi, dalle descrizioni e dalla nota cavalleresca segnata da Rustichello. Lo studioso conclude:

Forse si potrebbe spiegare con motivi storico-culturali la scelta del genere trattato, rispetto a una possibile narrazione in forma personale del viaggio e del soggiomo nell'impero gengiskhanide. La scelta non è comunque indenne da interessanti contraddizioni. Autobiografici sono infatti i primi 18 capitoli. (xxiv-xxv)

Non c'è alcun dubbio circa l'identità letteraria del libro di Marco Polo: "è un libro di geografia, non è un libro di avventure" ("Marco Polo" 15), ma Segre rileva che l'opera poliana, munita di illustrazioni, veniva solitamente intitolata Livre des Merveilles e faceva parte di raccolte di testi sull'Oriente ("Marco delle meraviglie" 95). È ovvio che quelle raccolte fantasiose e fantastiche (quali il ms. fr. 1116 della Bibliothèque Nationale di Parigi) venivano lette come libri di avventure, e così ci si può rifare alla teoria secondo cui il genere dell'opera va determinato in relazione al manoscritto, alla traduzione e all'ambiente; infatti all'inizio del XV secolo, quando fu copiato quel manoscritto, rientrano in circolazione quelle "fantasie sull'Oriente" che non avrebbero più avuto ragion d'essere dopo l'apparizione del libro del Veneziano ("Marco Polo" 19).

$\mathrm{Da}$ quanto affermato da Segre, si deduce che il Milione è soggetto a spostamenti e cambiamenti di genere a seconda del tipo di lettore, il che implica che non è possibile suggerire una collocazione definitiva dell'opera perché con tale tentativo si imporrebbero limiti alla fruizione del testo. Segre ha forse affrontato in modo leggermente diverso la questione facendo uso delle categorie del trattato geografico e dell'enciclopedia come se fossero distinte l'una dall'altra, ma, al tempo stesso, ha sottolineato quanto fosse significativa la presenza dell'opera poliana nelle raccolte meraviglioso-fantastiche. Il libro di Marco Polo risulta così onnipresente nella letteratura di tutti e plasmabile secondo gli interessi dei lettori.

Dal canto suo lo storico francese Jacques Heers afferma che l'opera poliana è un "ouvrage encyclopédique qui se propose essentiellement une description de l'Asie"; si tratta tuttavia di un caso "indéchiffrable" e "vouloir, par habitude, faire du Devisement dou monde un récit de voyage est manifestement une erreur" (125-26). Il testo non può essere definito itinerario perché si presenta "dans le plus complet désordre géographique" (130), ma si dovrebbe 
classificare piuttosto come "recueil de curiosités" (132) che pullula di "descriptions géographiques, analyses économiques, tableaux de géopolitique" (134) con procedimenti espositivi "toujours les mêmes, propres aux conteurs, aux écrivains de cour, où le mot "conter' revient à chaque occasion" (133), il che non esclude che l'opera abbia "la trame . . . d'un traité didactique" (135). La fortuna del testo si deve quindi ricondurre alle "cohabitations qui semblent indiquer une large diffusion du livre" (136). Heers ha messo in rilievo l'apporto del lettore: l'autore o, in sua vece, lo scriba, ha sempre avuto ben presente il destinatario dell'opera.

Tutte le caratteristiche relative alla letteratura di viaggi e di scoperte, "composta di generi e sottogeneri" (687), sono secondo Cardona "già in qualche modo sussunte in un testo di straordinaria fortuna, Le divisament dou monde" (690). La confluenza di elementi provenienti da varie tradizioni dimostra che l'autore intendeva essere quanto più possibile esauriente, il che allontana l'opera poliana dalla relazione di memorie personali e la avvicina al trattato geografico. In conclusione il Cardona rivolge un invito agli autori di storie della letteratura affinché essi accolgano la letteratura di viaggi, un genere dalla tipologia variatissima assai diffusa in tutta Europa.

Nell'introduzione della più recente edizione del Milione-titolo veramente emblematico "perché svariate sono le possibili 'chiavi di lettura"' (viii)-Ruggero M. Ruggieri mette in evidenza lo stretto nesso intercorrente tra l'opera poliana e il romanzo cavalleresco. In questa prospettiva (e numerosi sono stati nella storia della critica del libro i tentativi di paragone tra le opere originali di Rustichello e quella poliana) lo studioso si sofferma in particolare sui punti in comune tra il Milione e il Morgante. Tale prospettiva

che nulla intende detrarre alla realtà dei fatti e alla vissuta quotidianità della crona$\mathrm{ca}$, ben si addice all'atmosfera avventurosa e "meravigliosa" del tessuto narrativo, il quale cosi-forse grazie all'interessata collaborazione dell'amanuense coautore $\mathrm{Ru}$ stichello-viene improntata, in più di un episodio, ad un 'umanesimo cavalleresco' ... allargantesi a contorni e contenuti euro-asiatici. Non per nulla uno dei versantiquello europeo- offre termini di confronto e verifiche che si chiamano Chanson de Roland e Morgante, Intelligenza e Fatti de Spagna, Orlando Innamorato e Orlando Furioso. (viii-ix)

Se si pud stabilire un parallelo tra il Milione e le "cronache mongoliche" (il Ruggieri denomina in tal modo le opere dei Francescani che trattano di quella parte dell'Asia), risulterà chiaro che in esse "il rapporto tra la realtà, il meraviglioso e l'invenzione è di volta in volta diverso, diversamente dosato e diversamente interpretabile" (6), esattamente come avviene per il Milione che è interpretabile a più livelli. In tutte queste opere l'essenza, secondo il Ruggieri, deve essere ricondotta al "racconto dove l'elemento stupefacente o miracolistico, il thrilling e l'avventura, coesistono, senza dislivelli stilistici o narrativi, con episodi . . . notizie, ricordi e fatti per la prima volta accertati e descritti" (7). 
Nell'ambito della letteratura romanza il genere odeporico andava affermandosi e definendosi; ecco perché-in un tardo momento-il Milione e il Morgante, collocabili "in due ben distinti settori della produzione letteraria: da un lato il racconto 'storico' ... e dall'altro la finzione poetica", sono partecipi "di entrambi i 'generi', soprattutto quando svolgono la tematica ... dell'umanesimo cavalleresco", vale a dire quella tematica in cui "si compenetrano ... la lotta antisaracena e la graduale rivelazione (più o meno immaginosa e deformata) del mondo asiatico al mondo europeo" (12).

Nel Morgante il Ruggieri nota una "misteriosa e sottile (anche se talvolta nebulosa) atmosfera euro-asiatica" che è riconducibile alla realtà poliana: va riconosciuta al Pulci, "collezionista di vocaboli nuovi e strani, l'abilità di renderli evocatori e rivelatori di personaggi e paesi esotici, emergenti a tratti, e come d'incanto, da una realtà complessa e composita, Iontana e indeterminata" (23). Detta realtà è di puro stampo umanistico cavalleresco, di battaglie, di scontri, "di tenzoni armate ... tra interi popoli e singoli guerrieri, e in particolare tra cristiani e saraceni" (23-24). La maggior validità del discorso del Ruggieri si deve, decisamente, al parallelo "tra cristiani e saraceni da un lato . . . e tra mongoli e saraceni dall'altro" perché, e siamo d'accordo su questo, "equivale . . . ad una compenetrazione tra mondo europeo e mondo asiatico" (25).

Quel Riccardo Massano che doveva riprendere il lavoro iniziato dal Benedetto (cfr. Colesanti 2876) è autore di un profilo critico in cui Marco Polo viene definito "autentico scrittore ... che impone la sigla della sua personalità anzitutto nella struttura del libro", dettato da una "memoria di un'esperienza vissuta e indimenticabile, divenuta un tutt'uno con la sua personalità" (490-91). Quest'opera "divulgata e riassunta ed epilogata" è stata "anche declassata a guida pratica di informazione 'mercantile"' (491), nonostante fosse stata destinata al mondo dei dotti, come dimostra l'impostazione

tutta oggettiva e reale e scientifica-almeno nel senso medievale per cui scienza e arte ancora coincidevano - configurandosi in una serie successiva di 'quadri' che mirano a rivelare concretamente, pur con tutta la vaghezza che quei loci ancora mantenevano nei fantasiosi mappamondi medievali, le novitd e le meraviglie incontrate o comunque apprese entro quel mondo d'Asia, che l'immaginazione contemporanea popolava di mostri e di portenti. (492)

Il Milione-in cui si intravede l'elenco meccanico, la fissità delle formule e delle rubriche- "fa anche troppo sentire il limite di una stilizzazione compilatoria, meramente letteraria" dovuta sicuramente a Rustichello, a cui comunque si deve la forza di suggestione che ancora perdura ai nostri giomi.

Nel profilo tracciato dal Massano non si trova una presa di posizione ben chiara: pare che lo studioso non si sia voluto avventurare oltre i giudizi già noti. Il Ciccuto invece mette in evidenza la difficoltà di lettura relativa anche ad altre relazioni di viaggio, che hanno subíto:

sotto l'ideale crociato un processo di trans-codificazione anche profondo, che non 
poco ha contribuito del resto a incrementare le difficoltà già proprie a un 'genere letterario' in sede di definizione tecnica e di collocazione storica. (Crociata 221)

Il Milione non è stata l'unica opera odeporica sottoposta a cambiamenti stilistici e fors'anche contenutistici; lo stesso vale per altri testi manomessi da "copisti per passione e interpolatori pronti a deviare la significazione originaria del testo sui terreni della più tradizionale narratività fantastica" (224). Nel caso di Marco Polo si ha l'aggiunta dell'intervento ordinatore di Rustichello che porta a un "prodotto eccezionale di un genere ibrido, a metà strada fra il trattato di mercatura e il compendio enciclopedico" (228). Nonostante non si conosca la veste originaria stilistica letteraria del testo poliano, è stato gia comprovato che il numero delle edizioni manoscritte attesta la molteplicità delle letture compiute. Il libro di Marco Polo non va incasellato in un determinato gruppo; esso non appartiene ad una sola tradizione, ma costituisce un genere a sé stante. I due generi accennati dal Ciccuto inoltre comprendono una moltitudine di sottogeneri che definisce appieno l'essenza del Milione.

Con l'edizione del 1928 e i successivi studi benedettiani si è arrivati a una coesione testuale che ha incitato studiosi a intraprendere nuove indagini. Si vede che il libro di Marco Polo ha continuato ad essere uno dei più validi testi della letteratura di viaggi di ogni tempo e che, quantunque numerosi siano stati i tentativi in tal senso, non si è giunti finora a un accordo assoluto circa i generi letterari cui collegarlo. Svariate sono state le ipotesienciclopedia, trattato geografico, manuale di mercatura e via dicendo-ma come siamo venuti esponendo, l'opera poliana non va rinchiusa in nessuno in particolare; in essa si vede piuttosto l'accavallamento di generi ben diversi derivante dall'opera di manomissione svolta da chissà quanti intermediari, perché l'aspetto del libro non è stato definito e imposto soltanto dagli scribi, ma anche dai fruitori che a loro volta indussero i copisti a soddisfare i gusti degli utenti e del mercato.

\section{Brock University}

\section{NOTE}

1 Cfr. Segre, "Marco Polo: filologia".

2 Si veda inoltre Ruggieri, "Nota introduttiva" ix.

3 Dello stesso Tucci si confrontino pure "Marco Polo" e "Mercanti veneziani".

4 Todorov, citato dalla Bertolucci Pizzorusso ("Enunciazione" 19), scrive: "Au début et à la fin de chaque chapitre nous voyons apparaître ce sujet [le conte] ... " (150).

\section{OPERE CITATE}

Almagia, Roberto. La figura e l'opera di Marco Polo secondo recenti studi. Roma: Istituto Italiano per il Medio ed Estremo Oriente, 1938.

"A proposito di recenti studi su Marco Polo e i suoi viaggi". Rivista geografica italiana 62.2 (1955): 81-100. 
Badel, Pierre-Yves. "Lire la merveille selon Marco Polo". Revue des sciences humaines 4.183 (1981): 6-16.

Bertolucci Pizzorusso, Valeria. "Enunciazione e produzione del testo nel Milione". Studi mediolatini e volgari 25 (1977): 5-43.

"Prefazione". In Marco Polo. Milione. Versione toscana del Trecento. Milano: Adelphi, 1982. Seconda edizione. ix-xxi.

Borlandi, Franco. "Alle origini del libro di Marco Polo". In Studi in onore di Amintore Fanfani. Vol. 2. Milano: A. Giuffré, 1962. 105-147.

Camesasca, Ettore. "Introduzione". In Marco Polo. Il Milione. Novara: Istituto Geografico De Agostini, 1955. 5-32.

Cardona, Giorgio R. "I viaggi e le scoperte". In Letteratura italiana. Ed. Alberto Asor Rosa. Vol. 5. Le Questioni. Torino: Einaudi, 1986. 687-716.

Carile, Antonio. "Territorio e ambiente nel Divisament dou Monde di Marco Polo". Studi veneziani 1 (1977): 13-36.

Ciccuto, Marcello. "La crociata immaginaria di Marco Polo". Italianistica 16.2 (1987): 221-33. "Introduzione". In Marco Polo. Il Milione. Milano: Rizzoli, 1981. 11-45.

Colesanti, Massimo. "Luigi Foscolo Benedetto". In Letteratura italiana. I critici. Ed. Gianni Grana. Vol. 4. Milano: Marzorati, 1970. 2865-92.

Contini, Gianfranco. "Quando Marco Polo scopriva la Cina". Repubblica (20 aprile 1976).

Eco, Umberto. “Accidenti che giomalista!”. L'Espresso 47 (28 novembre 1982): 154-65.

Ferroni, Giulio. "Giorgio Manganelli". In Letteratura italiana. Novecento. I contemporanei. Ed. Gianni Grana. Vol. 10. Milano: Marzorati, 1979. 10057-81.

Giani, Renato. "Prefazione". In Marco Polo. Il Milione. Roma: Carlo Colombo, 1954. vIxxxiv.

Heers, Jacques. "De Marco Polo a Christophe Colomb: comment lire le Devisement dou monde?". Journal of Medieval History 10.2 (1984): 125-43.

Manganelli, Giorgio. "Letteratura fantastica". In La letteratura come menzogna. Milano: Feltrinelli, 1967. 44-50.

"Marco Polo". In Angosce di stile. Milano: Rizzoli, 1981. 162-73.

Massano, Riccardo. "Marco Polo 1254-1324". In Dizionario critico della letteratura italiana. Diretto da Vittore Branca. Vol. 3. Torino: UTET, 1986. Seconda edizione. 489-94.

Olschki, Leonardo. L'Asia di Marco Polo. Firenze: Sansoni, 1957.

Quaglio, Antonio E. "Retorica, prosa e narrativa del Duecento". In La letteratura italiana: Storia e testi. Direttore Carlo Muscetta. Il Duecento. Dalle Origini a Dante. Vol. 1. Tomo 2. Bari: Laterza, 1970. 257-428.

Roncaglia, Aurelio. "La letteratura franco-italiana". In Storia della letteratura italiana. Diretta da Emilio Cecchi e Natalino Sapegno. Vol. 2. Il Trecento. Milano: Garzanti, 1965. 727-59. Ruggieri, Ruggero M. “Introduzione”. In Marco Polo. Il Milione. Firenze: Olschki, 1986. 3-99. "Nota introduttiva". In Marco Polo. Il Milione. Firenze: Olschki, 1986. vii-xi. Scognamiglio, Gioacchino. "Saggio di bibliografia poliana". L'Italia che scrive 37.10 (1954): $143-48$.

Segre, Cesare. "Le forme e le tradizioni didattiche". In Grundriss der romanischen Literaturen des Mittelalters. Ed. Hans Robert Jauss. Vol. 6. Tomo 1. La littérature didactique, allegorique et satirique. Heidelberg: Carl Winter Universitätsverlag, 1968. 58-145.

"Introduzione". In Marco Polo. Milione. Le divisament dou monde. Il Milione nelle redazioni toscana e franco-italiana. Ed. Gabriella Ronchi. Milano: Mondadori, 1982. xi-xxix.

1963.

Lingua, stile e societd. Studi sulla storia della prosa italiana. Milano: Feltrinelli,

91-110.

"Marco delle meraviglie". FMR Mensile di Franco Maria Ricci 14 (1983):

"Marco Polo: filologia e industria culturale". In Avventure del "Milione". 
Parma: Edizioni Zara, 1983. 9-20.

Surdich, Francesco. "La più recente storiografia poliana". In Storiografia e storia. Studi in onore di Eugenio Dupre Theseider. Vol. 1. Roma: Bulzoni, 1974. 105-21.

Todorov, Tzvetan. Poétique de la prose. Paris: Editions du Seuil, 1971.

Tucci, Ugo. "Marco Polo, mercante". In Venezia e l'Oriente. Ed. Lanfranco Lanciotti. Firenze: Olschki, 1987. 323-37.

"Mercanti veneziani in Asia lungo l'itinerario poliano". In Venezia e l'Oriente. Ed. Lanfranco Lanciotti. Firenze: Olschki, 1987. 307-21.

"I primi viaggiatori e l'opera di Marco Polo". In Storia della cultura veneta.

Vol. 1. Dalle Origini al Trecento. Ed. Gianfranco Folena. Vicenza: Neri Pozza, 1976. 654-70. 